COMMUNICATIONS IN

ANALYSIS AND GEOMETRY

Volume 12, Number 3, 703-732, 2004

\title{
An Optimal Loewner-type Systolic Inequality and Harmonic One-forms of Constant Norm
}

\author{
ViCtor Bangert $^{1}$ AND Mikhail Katz ${ }^{2}$
}

\begin{abstract}
We present a new optimal systolic inequality for a closed Riemannian manifold $X$, which generalizes a number of earlier inequalities, including that of C. Loewner. We characterize the boundary case of equality in terms of the geometry of the Abel-Jacobi map, $\mathcal{A}_{X}$, of $X$. For an extremal metric, the map $\mathcal{A}_{X}$ turns out to be a Riemannian submersion with minimal fibers, onto a flat torus. We characterize the base of $\mathcal{A}_{X}$ in terms of an extremal problem for Euclidean lattices, studied by A.-M. Bergé and J. Martinet. Given a closed manifold $X$ that admits a submersion $F$ to its Jacobi torus $T^{b_{1}(X)}$, we construct all metrics on $X$ that realize equality in our inequality. While one can choose arbitrary metrics of fixed volume on the fibers of $F$, the horizontal space is chosen using a multi-parameter version of J. Moser's method of constructing volume-preserving flows.
\end{abstract}

\section{Introduction and statement of main theorems.}

We present a new optimal systolic inequality, which generalizes a number of earlier inequalities. These are the inequalities of C. Loewner [Pu52], J. Hebda [He86, Theorem A], certain results of G. Paternain [Pa01], and also inequality [BK03, Corollary 2.3]. Given a compact, oriented Riemannian manifold $X$ with positive first Betti number $b>0$, our optimal inequality (1.1) is a scale-invariant inequality providing a lower bound for the total volume of $X$, in terms of the product of its conformal 1-systole and its systole of codimension 1. The definitions of the systolic invariants involved appear in Section 2.

In [BK03, Corollary 2.3], the authors generalize J. Hebda's inequality (2.10) to arbitrary Betti number. In the present work, we strengthen the inequality by replacing the stable 1-systole by its conformal analogue.

\footnotetext{
${ }^{1}$ Partially Supported by DFG-Forschergruppe 'Nonlinear Partial Differential Equations: Theoretical and Numerical Analysis.'

${ }^{2}$ Supported by the Israel Science Foundation grants 620/00-10.0 and 84/03.
} 
Theorem 1.1. Every compact, orientable $n$-manifold $X$ with positive first Betti number $b=b_{1}(X, \mathbb{R})$ satisfies the inequality

$$
\operatorname{confsys}_{1}(g) \operatorname{sys}_{n-1}(g) \leq \gamma_{b}^{\prime} \operatorname{vol}_{n}(g)^{\frac{n-1}{n}}
$$

for every Riemannian metric $g$ on $X$.

The definition (3.1) of the Bergé-Martinet constant $\gamma_{b}^{\prime}$ and the associated dual-critical lattices, $c f$. Definition 3.1, implies that $\gamma_{b}^{\prime}$ is precisely the optimal constant in inequality (1.1) in the class of flat tori $\left(T^{b}, g\right)$. We also generalize Hebda's discussion of equality in inequality (2.10) as follows.

Theorem 1.2. Under the hypotheses of Theorem 1.1, one has equality in (1.1) if and only if there exists a dual-critical lattice $L \subset \mathbb{R}^{b}$ and a Riemannian submersion of $X$ onto the flat torus $\mathbb{R}^{b} / L$, such that all fibers are connected minimal submanifolds of $X$.

In particular, equality in (1.1) can only occur if $b_{1}(X, \mathbb{R}) \leq n$.

An alternative way of stating the conclusion of Theorem 1.2 is that we have equality in (1.1) if and only if the deck transformations of the Jacobi variety (4.1) form a dual-critical lattice, while the Abel-Jacobi map (4.2) is a Riemannian submersion with connected minimal fibers.

Example 1.3. Let $(X, g)$ be a Riemannian 4-manifold with first Betti number $b_{1}(X)=3$. For instance, $X$ could be the product of a circle by the nilmanifold given by the total space of the circle bundle over $T^{2}$ with nonzero Euler number. Then inequality (1.1) takes the form

$$
\operatorname{confsys}_{1}(g) \operatorname{sys}_{3}(g) \leq \sqrt{\frac{3}{2}} \operatorname{vol}_{4}(g)^{\frac{3}{4}}
$$

with equality possible if and only if the deck transformations of the Jacobi torus of $X$ form a cubic face-centered lattice, $c f$. Example 3.3. The possible topology of $X$ was clarified in [NV03], cf. Remark 1.4 below.

Note that $X$ admits a Riemannian submersion with minimal fibers to a flat $b$-dimensional torus if and only if all of its harmonic one-forms have pointwise constant norm, $c f$. [ES64] and Section 6. In Theorem 12.6 we characterize all the metrics satisfying the boundary case of equality in (1.1). We employ a multi-parameter generalisation of the method of J. Moser [Mo65] of constructing volume-preserving flows to determine all such metrics.

We study the relation between harmonic one-forms of constant norm and Riemannian submersions to a flat torus with minimal fibers, $c f$. Section 6 . 
Such submersions are special cases of foliations with minimal leaves that have been studied in the theory of foliations, cf. [Ru79], [Su79], [KT82], and the survey by V. Sergiescu [Se88]. However, the metrics in our context are much more special.

In the class of flat tori $T^{b}$, the discussion of equality reduces to an extremal problem for Euclidean lattices, studied by A.-M. Bergé and J. Martinet [BM89]. Namely, a flat torus $T^{b}$ realizes equality if its deck transformations form a so-called dual-critical lattice of rank $b$, cf. Definition 3.1. Thus, if $b=n$, then Theorem 1.2 implies that $(X, g)$ is a flat torus $\mathbb{R}^{n} / L$, for a suitable dual-critical lattice $L \subset \mathbb{R}^{n}$.

If $\operatorname{dim}(X)=b_{1}(X)$ and all harmonic 1-forms have constant norm, $\mathrm{G}$. Paternain [Pa01, Corollary 2] proved that $X$ is flat. The relation of the present work to [Pa01] is further clarified in Remark 6.4.

D. Kotschick [Ko01] defines the concept of formal Riemannian metric and proves, in particular, that for such a metric all harmonic forms (of arbitrary degree) have constant norm. Moreover, if $n=b$ then $M$ is flat [Ko01, Theorem 7], while in general one has $b \leq n$ [Ko01, Theorem 6]. Both of these statements parallel our discussion of equality in (1.1).

Remark 1.4. P. A. Nagy and C. Vernicos [NV03] study Riemannian manifolds $(X, g)$ with all harmonic one-forms of constant norm in the case $b_{1}(X)=\operatorname{dim} X-1$. Assuming that $X$ is compact, orientable and connected, they prove that $X$ is diffeomorphic to a two-step nilmanifold.

Systolic inequalities are metric-independent inequalities involving minimal volumes of homology (or 1-dimensional homotopy) classes in a Riemannian manifold. They have a certain similarity to the classical isoperimetric inequality. Over the past few years it has become clear that systolic inequalities are systematically violated as soon as an unstable $k$-systole with $k \geq 2$ is involved, $c f$. [Ka95, BabK98, BKS98, Bab02, Ka02].

On the other hand, a number of systolic inequalities, including those involving stable and conformal systoles, may be found in the survey [Gr96], as well as in the more recent works [BK03, Ka03]. See the recent survey [CK03, Figure 4.1] for a 2-D map of systolic geometry, which places such results in mutual relation.

Systolic inequalities with optimal constants are rare. In addition to the ones by C. Loewner (2.9) and J. Hebda (2.10), there are the inequalities by P.M. $\mathrm{Pu}[\mathrm{Pu} 52]$ for the projective plane, as well as [Bav86], [Sa88] for the Klein bottle, and [Gr99, pp. 259-260] for higher dimensional tori, $c f$. [CK03, inequality (5.14)]. A different optimal generalisation of Loewner's inequality 
as well as of the inequality implied in [Gr99, pp. 259-260], is studied in [IK04, BCIK1, BCIK2].

\section{Norms on (co-)homology and definition of systoles.}

In this section we assume that $X$ is a compact, oriented and connected manifold, $\operatorname{dim} X=n$, with Riemannian metric $g$. For $1 \leq p \leq \infty$ we define the $L^{p}$-norm \|\|$_{p}^{*}$ on $H^{k}(X, \mathbb{R})$ and the dual norms \|\|$_{p}$ on the dual space $H^{k}(X, \mathbb{R})^{*}=H_{k}(X, \mathbb{R})$. Then $k$-systoles of $(X, g)$ will be defined as the minima of such norms on non-zero elements in the integer lattice $H_{k}(X, \mathbb{Z})_{\mathbb{R}}$ in $H_{k}(X, \mathbb{R})$.

The $L^{p}$-norms on $H^{k}(X, \mathbb{R}), k \in\{0, \ldots, n\}$, are quotient norms of the corresponding norms on closed forms. For $\omega \in \Omega^{k} X$ and $1 \leq p<\infty$ we set

$$
\|\omega\|_{p}=\left(\int_{X}\left\|\omega_{x}\right\|^{p} d v o l_{n}(x)\right)^{\frac{1}{p}},
$$

where

$$
\left\|\omega_{x}\right\|=\max \left\{\omega_{x}\left(v_{1}, \ldots, v_{k}\right)|| v_{i} \mid \leq 1 \text { for } 1 \leq i \leq k\right\}
$$

is the pointwise comass norm of $\omega_{x}$. For $p=\infty$, we set

$$
\|\omega\|_{\infty}=\max _{x \in X}\left\|\omega_{x}\right\|
$$

In the cases $k=1$ and $k=n-1$ which are mainly of interest in the present paper, this comass norm coincides with the usual (Euclidean) norm $\left|\omega_{x}\right|$ induced by $g$. So, for 1 - or $(n-1)$-forms $\omega$, we will often use the symbol $|\omega|_{p}$ instead of $\|\omega\|_{p}$.

If $\alpha \in H^{k}(X, \mathbb{R})$ and $1 \leq p \leq \infty$, we define

$$
\|\alpha\|_{p}^{*}=\inf \left\{\|\omega\|_{p} \mid \omega \in \alpha\right\},
$$

where the expression $\omega \in \alpha$ means that $\omega$ is a smooth closed $k$-form representing $\alpha$. For $p=2$, Hodge theory implies that every cohomology class $\alpha$ contains a unique harmonic form $\omega$. If $k \in\{1, n-1\}$, this form $\omega$ is characterized by the equality $|\omega|_{2}=\|\alpha\|_{2}^{*}$. In particular, the norm \|\|$_{2}^{*}$ on $H^{1}(X, \mathbb{R})$ and $H^{n-1}(X, \mathbb{R})$ is induced by the $L^{2}$ scalar product $\langle$,$\rangle , on$ harmonic forms.

The non-degenerate Kronecker pairing

$$
[,]: H_{k}(X, \mathbb{R}) \times H^{k}(X, \mathbb{R}) \rightarrow \mathbb{R}
$$


induced by integration of closed forms over cycles, allows us to define a norm \|\|$_{p}$ on $H_{k}(X, \mathbb{R})$ dual to the $L^{p}$-norm \|\|$_{p}^{*}$ on $H^{k}(X, \mathbb{R})$. Thus, given $h \in H_{k}(X, \mathbb{R})$, we set

$$
\|h\|_{p}=\max \left\{[h, \alpha] \mid \alpha \in H^{k}(X, \mathbb{R}),\|\alpha\|_{p}^{*} \leq 1\right\} .
$$

Given a lattice $L$ in a finite-dimensional normed vector space $(V,\|\|)$, we denote by

$$
\lambda_{1}(L)=\lambda_{1}(L,\|\|)
$$

the minimal norm of a non-zero lattice vector. Then the stable $k$-systole $\operatorname{stsys}_{k}(g)$ is defined as follows:

$$
\operatorname{stsys}_{k}(g)=\lambda_{1}\left(H_{k}(X, \mathbb{Z})_{\mathbb{R}},\|\|_{\infty}\right) .
$$

Here $H_{k}(X, \mathbb{Z})_{\mathbb{R}}$ denotes the lattice of integral classes in $H_{k}(X, \mathbb{R})$.

The following geometric interpretation of the norm \|\|$_{\infty}$ is well known and not obvious, cf. [Fe74, 4.10 and 5.8] or [Gr99, 4.18 and 4.35]. Given an integer class $h \in H_{k}(X, \mathbb{Z})$, let $\operatorname{vol}_{k}(h)$ denote the infimum of the Riemannian volumes of all integer $k$-cycles representing $h$. Let $h_{\mathbb{R}}$ denote the class corresponding to $h$ in $H_{k}(X, \mathbb{R})$. H. Federer proved the equality

$$
\left\|h_{\mathbb{R}}\right\|_{\infty}=\lim _{i \rightarrow \infty} \frac{1}{i} \operatorname{vol}_{k}(i h),
$$

which is the origin of the term stable norm for \|\|$_{\infty}$.

One also has the (homological) $k$-systoles, denoted $s y s_{k}$ and defined by

$$
\operatorname{sys}_{k}(g)=\min \left\{\operatorname{vol}_{k}(h) \mid h \in H_{k}(X, \mathbb{Z}) \backslash\{0\}\right\} .
$$

If $H_{k}(X, \mathbb{Z})$ is torsion-free, then $\operatorname{stsys}_{k}(g) \leq \operatorname{sys}_{k}(g)$. It is a result due to H. Federer $[\mathrm{Fe} 74,5.10]$ that, in the assumption that $X$ is orientable, one has

$$
\|h\|_{\infty}=\operatorname{vol}_{n-1}(h) \text { for all } h \in H_{n-1}(X, \mathbb{Z}),
$$

and hence

$$
\operatorname{stsys}_{n-1}(g)=\operatorname{sys}_{n-1}(g) .
$$

It is not difficult to see that $\operatorname{sys}_{n-1}(g)$ is the infimum of the $(n-1)$-volumes of compact, orientable, non-separating hypersurfaces in $X$.

For $p=\frac{n}{k}$, the norms \|\|$_{p}^{*}$ on $H^{k}(X, \mathbb{R})$ and \|\|$_{p}$ on $H_{k}(X, \mathbb{R})$ are conformally invariant. Hence the quantity

$$
\operatorname{confsys}_{k}(g)=\lambda_{1}\left(H_{k}(X, \mathbb{Z})_{\mathbb{R}},\|\|_{\frac{n}{k}}\right)
$$


is conformally invariant, and is called the conformal $k$-systole of $(X, g)$. If $h \in H_{k}(X, \mathbb{R})$ then the quantity $\|h\|_{\frac{n}{k}}$ is the supremum of the stable norms of $h$ with respect to all metrics that are conformal to $g$ and have unit volume, cf. [Gr83, 7.4.A]. In particular, one has

$$
\operatorname{stsys}_{k}(g) \leq \operatorname{confsys}_{k}(g) \operatorname{vol}_{n}(g)^{\frac{k}{n}} .
$$

However, the supremum of $\operatorname{stsys}_{k}(\tilde{g})$ over all unit volume metrics $\tilde{g}$ conformal to $g$ may be smaller than confsys $_{k}(g)$. Indeed, the usual systole and the conformal systole obey dramatically different asymptotic bounds even in the case of surfaces. Thus, P. Buser and P. Sarnak [BS94] construct a sequence of surfaces $\Sigma_{s}$ of genus $s \rightarrow \infty$, satisfying an asymptotically infinite lower bound

$$
\operatorname{confsys}_{1}\left(\Sigma_{s}\right)^{2} \geq c \log s \text { as } s \rightarrow \infty,
$$

for a suitable constant $c>0$. An asymptotically vanishing upper bound

$$
\frac{\text { sys }_{1}\left(\Sigma_{s}\right)^{2}}{\operatorname{area}\left(\Sigma_{s}\right)} \leq C \frac{(\log s)^{2}}{s}
$$

appears in [Gr96]. Now we can state the known systolic inequalities that are generalized by Theorem 1.1.

Loewner's inequality. (see [Pu52]) Every riemannian metric $g$ on the torus $T^{2}$ satisfies

$$
\operatorname{sys}_{1}(g)^{2} \leq \frac{2}{\sqrt{3}} \operatorname{area}(g) \text {. }
$$

A metric satisfying the case of equality in (2.9) is homothetic to the quotient of $\mathbb{C}$ by the lattice spanned by the cube roots of unity.

Note that in the case of the 2-torus, the quantity $\operatorname{sys}_{1}(g)$ is the length of the shortest noncontractible loop in $\left(T^{2}, g\right)$. For higher genus surfaces, Loewner's inequality is satisfied by hyperelliptic conformal classes, and in particular by all genus 2 surfaces [KS04, KL04].

Hebda's inequality. ([He86, Theorem A $])$ Let $X$ be a compact, orientable, $n$-dimensional manifold with first Betti number $b_{1}(X, \mathbb{R})=1$. Then every Riemannian metric $g$ on $X$ satisfies

$$
\operatorname{stsys}_{1}(g) \operatorname{sys}_{n-1}(g) \leq \operatorname{vol}_{n}(g)
$$

with equality if and only if $(X, g)$ admits a Riemannian submersion with connected minimal fibers onto a circle. 
Note that inequality (1.1) is a generalization of (2.9), while (2.10) follows as a special case of inequality (2.8). Indeed, the first Bergé-Martinet constant $\gamma_{1}^{\prime}$ equals one, while $\gamma_{2}^{\prime}=\frac{2}{\sqrt{3}}, c f$. [BM89, Proposition 2.13] and Section 3.

\section{Dual-critical lattices of Bergé and Martinet.}

Theorem 1.1 involves a constant $\gamma_{b}^{\prime}$, called the Bergé-Martinet constant. It is defined by setting, in the notation of formula (2.1),

$$
\gamma_{b}^{\prime}=\sup \left\{\lambda_{1}(L) \lambda_{1}\left(L^{*}\right) \mid L \subseteq\left(\mathbb{R}^{b},||\right)\right\},
$$

where the supremum is extended over all lattices $L$ in $\mathbb{R}^{b}$ with a euclidean norm || and where $L^{*}$ denotes the lattice dual to $L$. The supremum defining $\gamma_{b}^{\prime}$ is attained, $c f$. [BM89].

Definition 3.1. A lattice $L$ realizing the supremum in formula (3.1) is called dual-critical.

Remark 3.2. The constants $\gamma_{b}^{\prime}$ and the dual-critical lattices in $\mathbb{R}^{b}$ are explicitly known for $b \leq 4, c f$. [BM89, Proposition 2.13]. In particular, we have $\gamma_{1}^{\prime}=1, \gamma_{2}^{\prime}=\frac{2}{\sqrt{3}}$.

Example 3.3. In dimension 3, the value of the Bergé-Martinet constant, $\gamma_{3}^{\prime}=\sqrt{\frac{3}{2}}=1.2247 \ldots$, is slightly below the Hermite constant $\gamma_{3}=2^{\frac{1}{3}}=$ $1.2599 \ldots$ It is attained by the face-centered cubic lattice, which is not isodual [MH73, p. 31], [BM89, Proposition 2.13(iii)], [CS94].

In general, the following facts are known about the constants $\gamma_{b}^{\prime}$ :

$$
\gamma_{b}^{\prime} \leq \gamma_{b} \leq \frac{2}{3} b \text { for all } b \geq 2
$$

and

$$
\frac{b}{2 \pi e}(1+o(1)) \leq \gamma_{b}^{\prime} \leq \frac{b}{\pi e}(1+o(1)) \text { as } b \rightarrow \infty,
$$

cf. [LLS90, pp. 334 and 337].

\section{Jacobi variety and Abel-Jacobi map.}

Note that the flat torus $\mathbb{R}^{b} / L$ in Theorem 1.2 is isometric to the Jacobi variety

$$
J_{1}(X)=H_{1}(X, \mathbb{R}) / H_{1}(X, \mathbb{Z})_{\mathbb{R}}
$$


of $X$, and the Riemannian submersion is the Abel-Jacobi map

$$
\mathcal{A}_{X}: X \rightarrow J_{1}(X)
$$

induced by the harmonic one-forms on $X$, originally introduced by A. Lichnerowicz [Li69], $c f$. [Gr99, 4.21].

More precisely, let $E$ be the space of harmonic 1-forms on $X$, with dual $E^{*}$ canonically identified with $H_{1}(X, \mathbb{R})$. By integrating an integral harmonic 1 -form along paths from a basepoint $x_{0} \in X$, we obtain a map to $\mathbb{R} / \mathbb{Z}=$ $S^{1}$. In order to define a map $X \rightarrow J_{1}(X)$ without choosing a basis for cohomology, we argue as follows. Let $x$ be a point in the universal cover $\widetilde{X}$ of $X$. Thus $x$ is represented by a point of $X$ together with a path $c$ from $x_{0}$ to it. By integrating along the path $c$, we obtain a linear form, $h \rightarrow \int_{c} h$, on $E$. We thus obtain a map $\tilde{X} \rightarrow E^{*}=H_{1}(X, \mathbb{R})$, which, furthermore, descends to a map

$$
\overline{\mathcal{A}}_{X}: \bar{X} \rightarrow E^{*}, \quad c \mapsto\left(h \mapsto \int_{c} h\right)
$$

where $\bar{X}$ is the universal free abelian cover. By passing to quotients, this map descends to the Abel-Jacobi map (4.2). The Abel-Jacobi map is unique up to translations of the Jacobi torus.

\section{Summary of the proofs.}

The proof of Theorem 1.1, which will be completed in Section 9, depends on two results. First we prove that for conjugate exponents $p \in[1, \infty]$ and $q$, the Poincaré duality map

$$
P D_{\mathbb{R}}:\left(H^{1}(X, \mathbb{R}),\|\|_{p}^{*}\right) \rightarrow\left(H_{n-1}(X, \mathbb{R}),\|\|_{q}\right)
$$

is an isometry, see Proposition 7.1. On the other hand, the Hölder inequality implies a chain of inequalities for the norms \|\|$_{p}^{*}$ on $H^{1}(X, \mathbb{R})$, see Proposition 8.1, and, dually, opposite inequalities for the norms \|\|$_{p}$ on $H_{1}(X, \mathbb{R})$. This allows us to reduce inequality (1.1) to the case $p=2$ where the norms are Euclidean, so that the definition (3.1) of $\gamma_{b}^{\prime}$ applies. If one has equality in $(1.1)$, and if $\alpha \in H^{1}(X, \mathbb{Z})_{\mathbb{R}}$ is a nonzero element of minimal $L^{2}$-norm, then the chain of inequalities for $\|\alpha\|_{p}^{*}$ reduces to equality for all $p$, and we conclude that the harmonic representative of $\alpha$ has constant norm.

Similarly, the $L^{2}$-dual of every nonzero $h \in H_{1}(X, \mathbb{Z})_{\mathbb{R}}$ of minimal dual $L^{2}$-norm is represented by a harmonic one-form of constant norm, as well. 
Using a result of Bergé and Martinet [BM89] on dual-critical lattices, we then prove that equality in (1.1) implies that all harmonic one-forms have constant norm. Finally, the Riemannian submersion is the Abel-Jacobi map $\mathcal{A}_{X}$ of formula (4.2), see Section 6 .

In the case $b_{1}(X)=1, \mathrm{~J}$. Hebda [He86] remarked already that equality in his inequality (2.10) implies the existence of a nonzero harmonic one-form with constant norm, and hence of a Riemannian submersion with minimal fibers over a circle.

Although Riemannian submersions over flat tori with minimal fibers are relatively simple objects, it is not immediately clear what choices one has in constructing all of them. In the case $b_{1}(X)=1$, J. Hebda [He86] describes the simplest class of examples, namely local Riemannian products. In Theorem 12.6, we present a construction that starts with a submersion $F: X \rightarrow T^{m}$ over a flat torus, and characterizes all Riemannian metrics on $X$ for which $F$ is a Riemannian submersion with minimal fibers.

More specifically, we can prescribe arbitrary metrics of fixed volume on the fibers. To determine the metric, one additionally has to choose a suitable horizontal distribution. This choice depends on the choice of a linear map from $\mathbb{R}^{m}$ into the Lie algebra of vertical vector fields on $X$ that preserve the volume elements of the fibers. If the fiber dimension, $\operatorname{dim} X-m$, is greater than one, this Lie algebra is infinite-dimensional. In particular, in this case the Riemannian metrics satisfying the case of equality in (1.1) are much more flexible than local Riemannian products.

Remark 5.1. There are compact manifolds that admit a submersion to the circle, but do not carry a Riemannian metric possessing a nontrivial local product structure, e.g. hyperbolic manifolds fibering over a circle. Thus, our construction yields new topological types of examples even in the case $b_{1}(X)=1$.

\section{Harmonic one-forms of constant norm and flat tori.}

Most of the material of this section appears in [ES64, pp. 127-128]. We include the proofs of Lemma 6.1 and Proposition 6.3 for convenience. The proofs are mostly straightforward calculations in local Riemannian geometry.

First we give a geometric characterisation of the existence of a nonzero harmonic one-form of constant (pointwise) norm on a Riemannian manifold $(X, g)$. 
Lemma 6.1. If $\theta \neq 0$ is a harmonic one-form of constant norm, then the leaves of the distribution $\operatorname{ker} \theta$ are minimal, and the vector field $V_{\theta}$ that is $g$-dual to $\theta$ has geodesic flow lines orthogonal to $\operatorname{ker} \theta$.

Conversely, given a transversely oriented foliation of $X$ by minimal hypersurfaces, such that the orthogonal foliation consists of geodesics, there exists a unique harmonic one-form $\theta$ with $|\theta|=1$, such that $\operatorname{ker} \theta$ is everywhere tangent to the foliation by minimal hypersurfaces, and such that $\theta$ is positive on the oriented normals to the leaves.

Proof. Since $d \theta=0$, we can locally find a primitive $f$ of $\theta$, i.e. $\quad d f=\theta$ and hence $\operatorname{grad} f=V_{\theta}$. The condition $d^{*} \theta=0$ translates into $\operatorname{div}(\operatorname{grad} f)=$ $\Delta f=0$. Since $\left|V_{\theta}\right|=|\theta|$ is constant, for all vector fields $W$ on $X$ we have

$$
0=g\left(\nabla_{W} V_{\theta}, V_{\theta}\right)=\operatorname{Hess}(f)\left(W, V_{\theta}\right)=g\left(W, \nabla_{V_{\theta}} V_{\theta}\right) .
$$

Hence $\nabla_{V_{\theta}} V_{\theta}=0$, i.e. the flow lines of $V_{\theta}$ are geodesics. Since $V_{\theta}$ is normal to the leaves, while $\left|V_{\theta}\right|$ is constant and $\nabla_{V_{\theta}} V_{\theta}=0$, the condition $\operatorname{div}\left(V_{\theta}\right)=0$ is equivalent to the fact that the mean curvature of the leaves of $\operatorname{ker}(\theta)$ vanishes.

Conversely, let $\theta$ be the one-form with $|\theta|=1$ that defines the given foliation by minimal hypersurfaces and is positive on the oriented normals. We want to prove that $\theta$ is closed. Since the orthogonal foliation is geodesic by assumption, we have $\nabla_{V_{\theta}} V_{\theta}=0$. If $h$ is a locally defined regular function whose level sets are leaves of $\operatorname{ker}(\theta)$, then $\operatorname{grad} h=\lambda V_{\theta}$ for some nowhere vanishing function $\lambda$. Then for all vector fields $W, Z$ we have

$$
\operatorname{Hess}(h)(W, Z)=W(\lambda) g\left(V_{\theta}, Z\right)+\lambda g\left(\nabla_{W} V_{\theta}, Z\right) .
$$

Hence the bilinear form

$$
(W, Z) \rightarrow g\left(\nabla_{W} V_{\theta}, Z\right)
$$

is symmetric if $W$ and $Z$ are orthogonal to $V_{\theta}$. Since $\nabla_{V_{\theta}} V_{\theta}=0$ and $0=$ $W\left(\left|V_{\theta}\right|^{2}\right)=2 g\left(\nabla_{W} V_{\theta}, V_{\theta}\right)$, we conclude that

$$
g\left(\nabla_{W} V_{\theta}, Z\right)=g\left(\nabla_{Z} V_{\theta}, W\right)
$$

for all vector fields $W, Z$. This implies $d \theta=0$. As above, we see that the minimality of the leaves, together with the conditions $\left|V_{\theta}\right|=1$ and $\nabla_{V_{\theta}} V_{\theta}=0$, imply $\operatorname{div}\left(V_{\theta}\right)=0$, and hence $d^{*} \theta=0$. 
Remark 6.2. If $\operatorname{dim} X=2$ and if $X$ carries a nonzero harmonic one-form $\theta$ of constant norm, then $X$ is flat and, in particular, every harmonic one-form has constant norm. This can be seen as follows. According to Lemma 6.1, the form $\theta$ induces two orthogonal foliations by geodesics. So the Gauss-Bonnet formula applied to small geodesic squares implies that $X$ is flat. Then the lift of a harmonic one-form on $X$ to the universal cover is constant.

Recall that a closed one-form is called integral if its integrals over arbitrary closed curves are integers.

Proposition 6.3. For Riemannian manifolds $(X, g)$ the following two properties are equivalent:

(i) There exists a flat torus $\left(T^{m}, \bar{g}\right)$ and a Riemannian submersion $F$ : $(X, g) \rightarrow\left(T^{m}, \bar{g}\right)$ with minimal fibers.

(ii) The set of harmonic one-forms of constant norm contains an m-dimensional vector space generated by integral one-forms.

Proof of the implication $(i) \Rightarrow(i i)$. Since $\bar{g}$ is flat, every harmonic oneform $\bar{\theta}$ on $\left(T^{m}, \bar{g}\right)$ has constant norm. Integral forms pull back to integral forms, and so it suffices to prove that $F^{*} \bar{\theta}$ is a harmonic one-form of norm $\left|F^{*} \bar{\theta}\right|=|\bar{\theta}|$. This equality is true since $F$ is a Riemannian submersion. To show that $F^{*} \bar{\theta}$ is harmonic, note that $F^{*} \bar{\theta}$ is closed and of constant norm. This implies that the foliation orthogonal to $\operatorname{ker}\left(F^{*} \bar{\theta}\right)$ is geodesic if $\bar{\theta} \neq 0, c f$. the proof of Lemma 6.1. By Lemma 6.1, it remains to show that the leaves of the foliation $\operatorname{ker}\left(F^{*} \bar{\theta}\right)$ are minimal. This is a consequence of the minimality of the fibers of $F$, together with the fact that the leaves of $\operatorname{ker}\left(F^{*} \bar{\theta}\right)$ are geodesic in horizontal directions. Namely, if $v \in \operatorname{ker}\left(\left.F^{*} \bar{\theta}\right|_{x}\right)$ is orthogonal to the fiber $F_{x}=F^{-1}(F(x))$ through $x$ and if $c$ is the geodesic in $X$ with $\dot{c}(0)=v$, then $\dot{c}(t) \in \operatorname{ker}\left(\left.F^{*} \bar{\theta}\right|_{c(t)}\right)$ for all $t$.

Proof of the implication $(i i) \Rightarrow(i)$. Let $\theta_{1}, \ldots, \theta_{m}$ be linearly independent integral harmonic one-forms on $X$, such that all linear combinations $\sum_{i=1}^{m} r_{i} \theta_{i}$ with $r_{i} \in \mathbb{R}$ have constant norm. Note that this last property is equivalent to the constancy of all scalar products $g^{*}\left(\theta_{i}, \theta_{j}\right)$ with respect to the dual metric $g^{*}$. Let $\left(\bar{g}_{i j}\right)$ denote the matrix inverse to $\left(g^{*}\left(\theta_{i}, \theta_{j}\right)\right)$. Since the $\theta_{i}$ are integral, there exist functions $F_{i}: X \rightarrow \mathbb{R} / \mathbb{Z}$ such that $d F_{i}=\theta_{i}$. Then 
the map

$$
F=\left(F_{1}, \ldots, F_{m}\right): X \rightarrow \mathbb{R}^{m} / \mathbb{Z}^{m}
$$

is a Riemannian submersion from $(X, g)$ to $\mathbb{R}^{m} / \mathbb{Z}^{m}$ endowed with the constant metric $\bar{g}=\sum \bar{g}_{i j} d x_{i} \otimes d x_{j}$. It remains to prove that the fibers of $F$ are minimal. We can find a global orthonormal frame field $V_{1}, \ldots, V_{m}$ for the horizontal bundle $\mathcal{H}=\operatorname{ker}(D F)^{\perp} \subseteq T X$ by taking the $g$-dual of appropriate constant linear combinations of $\theta_{1}, \ldots, \theta_{m}$. Locally we can complete $V_{1}, \ldots, V_{m}$ to an orthonormal frame field $V_{1}, \ldots, V_{n}$ for $T X$. Since $V_{1}, \ldots, V_{m}$ are $g$-dual to harmonic one-forms, we know that

$$
\sum_{j=1}^{n} g\left(\nabla_{V_{j}} V_{i}, V_{j}\right)=\operatorname{div} V_{i}=0
$$

for $1 \leq i \leq m$, $c f$. the proof of Lemma 6.1. On the other hand, Lemma 6.1 implies $\nabla_{V_{j}} V_{j}=0$ for $1 \leq j \leq m$, and hence

$$
g\left(\nabla_{V_{j}} V_{i}, V_{j}\right)=V_{j}\left(g\left(V_{i}, V_{j}\right)\right)=0
$$

for $1 \leq j \leq m, 1 \leq i \leq n$. The preceding equations imply that

$$
\sum_{j=m+1}^{n} g\left(\nabla_{V_{j}} V_{i}, V_{j}\right)=0
$$

for $1 \leq i \leq m$. Since $V_{1}, \ldots, V_{m}$ are orthogonal to the fibers of $F$, this says that the mean curvature vector of the fibers of $F$ vanishes.

Remark 6.4. In the case $m=\operatorname{dim}(X)$, the statement of Proposition 6.3 also follows from the arguments employed by G. Paternain to prove [Pa01, Corollary 2]. These arguments are global and use the solution of the E. Hopf conjecture by D. Burago and S. Ivanov. They do not apply in the case $m<\operatorname{dim}(X)$.

In the corollary below, we show that a Riemannian submersion $F$ as in Proposition 6.3(i) is uniquely determined by the metric $g$ on $X$ and by the induced map $F_{*}: H_{1}(X, \mathbb{Z}) \rightarrow H_{1}\left(T^{m}, \mathbb{Z}\right)$.

Corollary 6.5. Suppose $I:\left(X_{0}, g_{0}\right) \rightarrow\left(X_{1}, g_{1}\right)$ is an isometry and for $i=0,1$ we are given Riemannian submersions $F_{i}$ with minimal fibers from $\left(X_{i}, g_{i}\right)$ to flat tori $\left(T^{m}, \bar{g}_{i}\right)$. If there exists an automorphism $A$ of $H_{1}\left(T^{m}, \mathbb{Z}\right)$ such that $\left(F_{1} \circ I\right)_{*}=A \circ\left(F_{0}\right)_{*}$ then there exists an isometry $\bar{I}=\left(T^{m}, \bar{g}_{0}\right) \rightarrow$ $\left(T^{m}, \bar{g}_{1}\right)$ such that $\bar{I} \circ F_{0}=F_{1} \circ I$. 


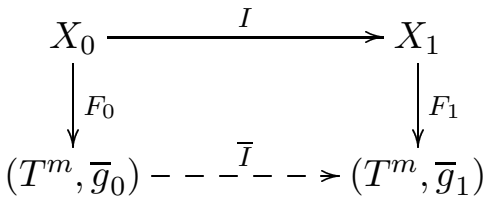

Figure 1: Construction of isometry $\bar{I}$

Proof. Let $L_{i} \subseteq \mathbb{R}^{m}$ be the lattice of deck transformations of $\left(T^{m}, \bar{g}_{i}\right)$. Then we can identify $A$ with a linear map $J: \mathbb{R}^{m} \rightarrow \mathbb{R}^{m}$ such that $J\left(L_{0}\right)=L_{1}$. If

$$
\bar{J}: \mathbb{R}^{m} / L_{0} \rightarrow \mathbb{R}^{m} / L_{1}
$$

denotes the diffeomorphism induced by $J$, then our assumption implies that $\left(F_{1} \circ I\right)_{*}=\left(\bar{J} \circ F_{0}\right)_{*}$. Hence, if $\theta$ is a constant one-form on $\mathbb{R}^{m} / L_{1}$, then the closed one-forms $\left(F_{1} \circ I\right)^{*} \theta$ and $\left(\bar{J} \circ F_{0}\right)^{*} \theta$ are cohomologous. From the proof of Proposition 6.3, we know that $\left(F_{1} \circ I\right)^{*} \theta$ and $\left(\bar{J} \circ F_{0}\right)^{*} \theta$ are harmonic with respect to $g_{0}$. By the uniqueness of the harmonic representative of a cohomology class, we conclude that

$$
\left(F_{1} \circ I\right)^{*} \bar{\theta}=\left(\bar{J} \circ F_{0}\right)^{*} \bar{\theta}
$$

for every constant one-form on $\mathbb{R}^{m} / L_{1}$. This implies the existence of $v \in$ $\mathbb{R}^{m} / L_{1}$ such that

$$
F_{1} \circ I=\bar{J} \circ F_{0}+v \text {. }
$$

If we define $\bar{I}: \mathbb{R}^{m} / L_{0} \rightarrow \mathbb{R}^{m} / L_{1}$ by $\bar{I}(y)=\bar{J}(y)+v$, then $\bar{I}$ satisfies $F_{1} \circ I=\bar{I} \circ F_{0}$, as illustrated in Figure 1. The map $\bar{I}$ is a diffeomorphism and a (local) isometry since $I$ is an isometry, and $F_{0}$ and $F_{1}$ are Riemannian submersions.

\section{Norm duality and the cup product.}

In this section, we assume that $(X, g)$ is a compact, $n$-dimensional, oriented, and connected Riemannian manifold. For $k \in\{1, n-1\}$ we consider the norms \|\|$_{p}^{*}$ on $H^{k}(X, \mathbb{R})$ and \|\|$_{p}$ on $H_{k}(X, \mathbb{R})$ defined in Section 2.

In Proposition 7.1, we prove that for every pair of conjugate exponents $p \in[1, \infty]$ and $q$, the Poincaré duality map is an isometry between $\left(H^{1}(X, \mathbb{R}),\|\|_{p}\right)$ and $\left(H_{n-1}(X, \mathbb{R}),\|\|_{q}\right)$, respectively between $\left(H^{n-1}(X, \mathbb{R}),\|\|_{p}\right)$ and $\left(H_{1}(X, \mathbb{R}),\|\|_{q}\right)$.

The results of the present section will be used in Sections 9 and 11 to prove the systolic inequality in Theorem 1.1 and to analyze the case of 
equality. For completeness and future reference, some of them are proved in greater generality than necessary for these applications.

Proposition 7.1. Let $p \in[1, \infty]$ and $q$ be conjugate exponents. Then the normed spaces $\left(H^{1}(X, \mathbb{R}),\|\|_{p}^{*}\right)$ and $\left(H^{n-1}(X, \mathbb{R}),\|\|_{q}^{*}\right)$ are dual to each other with respect to the cup product.

Remark 7.2. The claim of Proposition 7.1 is equivalent to either of the following two statements:

1. The Poincaré duality map

$$
P D_{\mathbb{R}}:\left(H^{1}(X, \mathbb{R}),\|\|_{p}^{*}\right) \rightarrow\left(H_{n-1}(X, \mathbb{R}),\|\|_{q}\right)
$$

is an isometry.

2. The Poincaré duality map

$$
P D_{\mathbb{R}}:\left(H^{n-1}(X, \mathbb{R}),\|\|_{q}^{*}\right) \rightarrow\left(H_{1}(X, \mathbb{R}),\|\|_{p}\right)
$$

is an isometry.

Proof of Proposition 7.1. A key idea is the application of the Hodge star operator in a suitable measurable context, $c f$. formula (7.3). Let $\alpha \in H^{1}(X, \mathbb{R})$, $\beta \in H^{n-1}(X, \mathbb{R})$. If $\omega$ (respectively, $\pi$ ) is a closed form representing $\alpha$ (respectively, $\beta$ ), then the cup product $\alpha \cup \beta$ satisfies

$$
P D_{\mathbb{R}}(\alpha \cup \beta)=\int_{X} \omega \wedge \pi
$$

Since one- and $(n-1)$-forms take values in the set of simple covectors, we can use $[\mathrm{Fe} 69$, p. 32] and the Hölder inequality to estimate

$$
\left|\int_{X} \omega \wedge \pi\right| \leq \int_{X}\left|\omega_{x}\right|\left|\pi_{x}\right| \operatorname{dvol}_{n}(x) \leq|\omega|_{p}|\pi|_{q}
$$

This proves

$$
P D_{\mathbb{R}}(\alpha \cup \beta) \leq\|\alpha\|_{p}^{*}\|\beta\|_{q}^{*}
$$

It remains to show that for every $\alpha \in H^{1}(X, \mathbb{R}) \backslash\{0\}$, there exists $\beta \in$ $H^{n-1}(X, \mathbb{R}) \backslash\{0\}$ such that

$$
P D_{\mathbb{R}}(\alpha \cup \beta)=\|\alpha\|_{p}^{*}\|\beta\|_{q}^{*},
$$


or, conversely, that for every $\beta \in H^{n-1}(X, \mathbb{R}) \backslash\{0\}$, there exists $\alpha \in$ $H^{1}(X, \mathbb{R}) \backslash\{0\}$ such that $(7.2)$ holds. Assume first that $2 \leq p<\infty$. It is shown in [Si70, Proof of Lemma 4.2] that there exists a one-form $\omega$ in the $L^{p}$-closure of $\alpha$ such that $|\omega|_{p}=\|\alpha\|_{p}^{*}$. It follows from [Ha92, Theorem 5.1] that the form

$$
\pi=|\omega|^{p-2}(* \omega)
$$

is weakly closed and determines a class $\beta \in H^{n-1}(X, \mathbb{R})$ such that $\|\beta\|_{q}^{*}=$ $|\pi|_{q}$. This implies $0 \neq \alpha \cup \beta=\|\alpha\|_{p}^{*}\|\beta\|_{q}^{*}$.

If $1<p<2$ and hence $2<q<\infty$, we can apply analogous arguments starting with an arbitrary $\beta \in H^{n-1}(X, \mathbb{R}) \backslash\{0\}$. We then obtain $\alpha \in$ $H^{1}(X, \mathbb{R}) \backslash\{0\}$ such that $(7.2)$ holds. Finally we treat the case $p=\infty$ and $q=1$. The proof in the case $p=1$ and $q=\infty$ is completely analogous. According to Remark 7.2, it suffices to prove that the map

$$
P D_{\mathbb{R}}:\left(H^{n-1}(X, \mathbb{R}),\|\|_{1}^{*}\right) \rightarrow\left(H_{1}(X, \mathbb{R}),\|\|_{\infty}\right)
$$

is an isometry. From (7.1) we conclude that $\left\|P D_{\mathbb{R}}(\beta)\right\|_{\infty} \leq\|\beta\|_{1}^{*}$ for every $\beta \in H^{n-1}(X, \mathbb{R})$. To prove the opposite inequality, we use some elementary facts from geometric measure theory. Recall that a closed normal one-current is a linear functional $T: \Omega^{1} X \rightarrow \mathbb{R}$ such that $T(d f)=0$ for every $f \in$ $C^{\infty}(M, \mathbb{R})$ and such that its mass $\mathbf{M}(T)$, defined by

$$
\mathbf{M}(T)=\sup \left\{\left.T(\omega)\left|\omega \in \Omega^{1}(M),\right| \omega\right|_{\infty} \leq 1\right\}
$$

is finite. Every closed normal one-current $T$ determines a homology class $[T] \in H_{1}(X, \mathbb{R})$ such that $[[T], \alpha]=T(\omega)$ whenever $\omega \in \alpha$. Every closed $(n-1)$-form $\pi$ defines a closed normal one-current $T_{\pi}$ by setting

$$
T_{\pi}(\omega)=\int_{X} \omega \wedge \pi
$$

Such one-currents are called smooth. One easily sees that

$$
\mathbf{M}\left(T_{\pi}\right)=|\pi|_{1}
$$

and

$$
P D_{\mathbb{R}}(\pi)= \pm\left[T_{\pi}\right]
$$

Functional analysis implies that for every $h \in H_{1}(X, \mathbb{R})$, there exists a closed normal one-current $T$ such that $[T]=h$ and $\mathbf{M}(T)=\|h\|_{\infty}, c f . \quad[\mathrm{Fe} 74$, Section 3]. 
We can smooth this $T$, cf. [Fe74, 4.1.18], and obtain a sequence $\pi_{i} \in$ $P D_{\mathbb{R}}^{-1}(h)$ such that

$$
\lim _{i \rightarrow \infty}\left|\pi_{i}\right|_{1}=\lim _{i \rightarrow \infty} \mathbf{M}\left(T_{\pi_{i}}\right)=\mathbf{M}(T)=\|h\|_{\infty} .
$$

This implies $\left\|P D_{\mathbb{R}}^{-1}(h)\right\|_{1}^{*} \leq\|h\|_{\infty}$.

\section{Hölder inequality in cohomology and case of equality.}

The Hölder inequality implies inequalities between the $L^{p}$ norms \|\|$_{p}$ for different values of $p$. We will prove that the harmonic representative of a cohomology class $\alpha \in H^{1}(X, \mathbb{R})$ has constant norm if and only if for some (and hence for every) $p \neq 2$, the inequality relating the $L^{2}$ - and the $L^{p}$-norm is an equality at $\alpha$. For $p=\infty$, this follows from [Pa01, Theorem $\mathrm{C}$ ].

Proposition 8.1. For every $\alpha \in H^{1}(X, \mathbb{R})$, the function

$$
p \in[1, \infty] \rightarrow\|\alpha\|_{p}^{*} \operatorname{vol}_{n}(g)^{-\frac{1}{p}}
$$

is weakly increasing. For $p \in[1, \infty] \backslash\{2\}$, the equality $\|\alpha\|_{p}^{*} \operatorname{vol}_{n}(g)^{-\frac{1}{p}}=$ $\|\alpha\|_{2}^{*} \operatorname{vol}_{n}(g)^{-\frac{1}{2}}$ holds if and only if the harmonic representative of $\alpha$ has constant norm.

Proof. The monotonicity follows from Hölder inequality. Next, we assume that the harmonic representative $\omega$ of $\alpha$ has constant norm. Due to the monotonicity, it suffices to prove that

$$
\|\alpha\|_{\infty}^{*} \leq\|\alpha\|_{2}^{*} \operatorname{vol}_{n}(g)^{-\frac{1}{2}} \leq\|\alpha\|_{1}^{*} \operatorname{vol}_{n}(g)^{-1}
$$

The first inequality follows directly from the constancy of $\left|\omega_{x}\right|$. To prove the second inequality, let $\left(\omega_{i}\right)_{i \in \mathbb{N}}$ be a sequence of representatives of $\alpha$ such that $\lim _{i \rightarrow \infty}\left|\omega_{i}\right|_{1}=\|\alpha\|_{1}^{*}$. Since $\omega_{i}-\omega$ is exact and $\omega$ is harmonic, we see that $\left\langle\omega_{i}-\omega, \omega\right\rangle=0$. Since $\left|\omega_{x}\right|$ is constant, we conclude that

$$
|\omega|_{2}^{2}=\left\langle\omega_{i}, \omega\right\rangle_{2} \leq\left|\omega_{i}\right|_{1}|\omega|_{2} \operatorname{vol}_{n}(g)^{-\frac{1}{2}}
$$

and hence

$$
\|\alpha\|_{1}^{*} \operatorname{vol}_{n}(g)^{-\frac{1}{2}} \geq\|\alpha\|_{2}^{*} .
$$

Finally, we assume that $p \in[1, \infty] \backslash\{2\}$ and that

$$
\left.\|\alpha\|_{p}^{*} \operatorname{vol}_{n}(g)^{-\frac{1}{p}}=\|\alpha\|_{2}^{*} \operatorname{vol}_{(} n\right)^{-\frac{1}{2}}
$$


and prove that the harmonic representative $\omega$ of $\alpha$ has constant norm. Due to the monotonicity, it suffices to treat the cases $1<p<2$ and $2<p<\infty$. If $1<p<2$, the discussion of equality in the Hölder inequality implies that $\omega$ has constant norm. If $2<p<\infty$, then there exists a one-form $\omega$ in the $L^{p}$-closure of $\alpha$ such that $|\omega|_{p}=\|\alpha\|_{p}^{*}, c f$. [Si70, proof of Lemma 4.2]. Now the Hölder inequality and (8.1) imply

$$
\begin{aligned}
|\omega|_{2} \operatorname{vol}_{n}(g)^{-\frac{1}{2}} & \leq|\omega|_{p} \operatorname{vol}_{n}(g)^{-\frac{1}{p}} \\
& =\|\alpha\|_{p}^{*} \operatorname{vol}_{n}(g)^{-\frac{1}{p}} \\
& =\|\alpha\|_{2}^{*} \operatorname{vol}_{n}(g)^{-\frac{1}{2}} .
\end{aligned}
$$

Hence $\omega$ is the harmonic representative of $\alpha$, the first inequality is an equality, and the norm of $\omega$ is constant.

Let $\langle,\rangle_{2}^{*}$ denote the scalar product on $H^{1}(X, \mathbb{R})$, with norm \|\|$_{2}^{*}$. Thus, if we identify $H^{1}(X, \mathbb{R})$ with the space of harmonic one-forms, then $\langle,\rangle_{2}^{*}$ corresponds to the $L^{2}$-scalar product. We define an isomorphism $I: H_{1}(X, \mathbb{R}) \rightarrow H^{1}(X, \mathbb{R})$ by

$$
\langle I(h), \alpha\rangle_{L^{2}}^{*}=[h, \alpha] \quad \text { for } h \in H_{1}(X, \mathbb{R}), \alpha \in H^{1}(X, \mathbb{R}) .
$$

Note that $\|h\|_{2}=\|I(h)\|_{2}^{*}$ for all $h \in H_{1}(X, \mathbb{R})$. Proposition 8.1 implies the following corollary.

Corollary 8.2. For every $h \in H_{1}(X, \mathbb{R})$, the function

$$
p \in[1, \infty] \rightarrow\|h\|_{p} \operatorname{vol}_{n}(g)^{\frac{1}{p}}
$$

is (weakly) decreasing. For $p \in[1, \infty] \backslash\{2\}$, the equality $\|h\|_{p} \operatorname{vol}_{n}(g)^{\frac{1}{p}}=$ $\|h\|_{2} \operatorname{vol}_{n}(g)^{\frac{1}{2}}$ holds if and only if the harmonic representative of $I(h)$ has constant norm.

Similarly, we can combine Propositions 7.1 and 8.1 to obtain the following corollary.

Corollary 8.3. For every $k \in H_{n-1}(X, \mathbb{R})$, the function

$$
p \in[1, \infty] \rightarrow\|k\|_{p} \operatorname{vol}_{n}(g)^{\frac{1}{p}}
$$

is (weakly) decreasing. For $p \in[1, \infty] \backslash\{2\}$, the equality $\|k\|_{p} \operatorname{vol}_{n}(g)^{\frac{1}{p}}=$ $\|k\|_{2} \operatorname{vol}_{n}(g)^{\frac{1}{2}}$ holds if and only if the harmonic representative of $P D_{\mathbb{R}}^{-1}(k)$ has constant norm. 


\section{Proof of Theorem 1.1.}

We assume that $(X, g)$ is a compact, oriented, and connected Riemannian manifold with $b=b_{1}(X, \mathbb{R}) \geq 1$. We first show how the results proved in Sections 7 and 8 imply Theorem 1.1:

$$
\operatorname{confsys}_{1}(g) \operatorname{sys}_{n-1}(g) \leq \gamma_{b}^{\prime} \operatorname{vol}_{n}(g)^{\frac{n-1}{n}},
$$

where $n=\operatorname{dim}(X)$ and $\gamma_{b}^{\prime}$ is the Bergé-Martinet constant. Note that inequality (9.1) is true also for disconnected manifolds once we know (9.1) for connected ones.

Proof of Theorem 1.1. The Euclidean vector spaces $\left(H_{1}(X, \mathbb{R}),\|\|_{2}\right)$ and $\left(H_{n-1}(X, \mathbb{R}),\|\|_{2}\right)$ are dual to each other with respect to the intersection pairing defined by

$$
h \cdot k=\left[h, P D_{\mathbb{R}}^{-1}(k)\right] .
$$

This follows from the fact that the map $P D_{\mathbb{R}}:\left(H^{1}(X, \mathbb{R}),\|\|_{2}^{*}\right) \rightarrow$ $\left(H_{n-1}(X, \mathbb{R}),\|\|_{2}\right)$ is an isometry, $c f$. Remark 7.2. Moreover, the pair of lattices $H_{1}(X, \mathbb{Z})_{\mathbb{R}} \subset H_{1}(X, \mathbb{R})$ and $H_{n-1}(X, \mathbb{Z})_{\mathbb{R}} \subset H_{n-1}(X, \mathbb{R})$ are dual to each other with respect to this pairing, $c f$. [BK03, Section 3]. Hence, formula (3.1) for the Bergé-Martinet constant $\gamma_{b}^{\prime}$ implies that

$$
\lambda_{1}\left(H_{1}(X, \mathbb{Z})_{\mathbb{R}},\|\|_{2}\right) \lambda_{1}\left(H_{n-1}(X, \mathbb{Z})_{\mathbb{R}},\|\|_{2}\right) \leq \gamma_{b}^{\prime},
$$

where $b=b_{1}(X)$. Invoking Corollaries 8.2 and 8.3, we obtain for all $p, p^{\prime} \in$ $[2, \infty]$ :

$$
\lambda_{1}\left(H_{1}(X, \mathbb{Z})_{\mathbb{R}},\|\|_{p}\right) \lambda_{1}\left(H_{n-1}(X, \mathbb{Z})_{\mathbb{R}},\|\|_{p^{\prime}}\right) \leq \gamma_{b}^{\prime} \operatorname{vol}_{n}(g)^{1-\frac{1}{p}-\frac{1}{p^{\prime}}}
$$

Now we specify parameter values to $p=n$ and $p^{\prime}=\infty$. Then inequality (9.2) specifies to (9.1), cf. definition of confsys 1 in formula (2.7), as well as (2.2) and (2.6).

Note that the inequality $\|\alpha\|_{L^{2}}^{*}\left(\operatorname{vol}_{n}(g)\right)^{\frac{1}{2}} \geq\left\|P D_{\mathbb{R}} \alpha\right\|_{\infty}$ for a class $\alpha \in$ $H^{1}(X, \mathbb{R})$ can also be proved more directly, as follows. Let $\alpha \in H^{1}(X, \mathbb{Z})_{\mathbb{R}}$ be a nonzero element in the integer lattice, and let $\omega \in \alpha$ be the harmonic 1 -form. Then there exists a map $f: X \rightarrow S^{1}=\mathbb{R} / \mathbb{Z}$ such that $d f=\omega$. Using the Cauchy-Schwartz inequality and the coarea formula, cf. [Fe69, 3.2.11], 
[Ch93, p. 267], we obtain

$$
\begin{aligned}
\|\alpha\|_{L^{2}}^{*}\left(\operatorname{vol}_{n}(g)\right)^{1 / 2} & =|\omega|_{2}\left(\operatorname{vol}_{n}(g)\right)^{1 / 2} \\
& \geq \int_{X}|d f| d v o l_{n} \\
& =\int_{S^{1}} \operatorname{vol}_{n-1}\left(f^{-1}(t)\right) d t \\
& \geq\|P D(\alpha)\|_{\infty},
\end{aligned}
$$

since the hypersurface $f^{-1}(t)$ is Poincaré dual to $\alpha$ for every regular value of $t$.

\section{Consequences of equality and criterion of dual-perfection.}

We now collect some consequences of equality in (9.1). From Corollaries 8.2 and 8.3, combined with Remark 6.2 (if $n=2$ ), we obtain Lemma 10.2 below.

Definition 10.1. A vector $v \in(L,\|\|)$ is called short if $\|v\|=\lambda_{1}(L,\|\|)$.

Lemma 10.2. Assume equality holds in (9.1). Then

1. if $\alpha \in\left(H^{1}(X, \mathbb{Z})_{\mathbb{R}},\|\|_{2}^{*}\right)$ is short, then the harmonic representative of $\alpha$ has constant norm;

2. if $h \in\left(H_{1}(X, \mathbb{Z})_{\mathbb{R}},\|\|_{2}\right)$ is short, then the harmonic representative of $I(h) \in H^{1}(X, \mathbb{R})$ as in (8.2) has constant norm.

Given a pair of dual lattices $L$ and $L^{*}$ in Euclidean space $\left(E,\|\|_{2}\right)$, consider the sets of short vectors

$$
\begin{aligned}
S(L) & =\left\{\alpha \in L \mid\|\alpha\|_{2}=\lambda_{1}(L)\right\}, \\
S\left(L^{*}\right) & =\left\{\beta \in L^{*} \mid\|\beta\|_{2}=\lambda_{1}\left(L^{*}\right)\right\},
\end{aligned}
$$

so that $S(L) \cup S\left(L^{*}\right) \subset E$. Let $Q(E)$ denote the vector space of symmetric bilinear forms on $E$, and let $\varphi: E \rightarrow Q(E)^{*}$ be the map defined by

$$
\varphi(\gamma)(q)=q(\gamma, \gamma)
$$

for $\gamma \in E$ and $q \in Q(E)$.

Definition 10.3 ([BM89]). A pair $\left(L, L^{*}\right)$ of dual lattices in Euclidean space is called dual-perfect if the set $\varphi\left(S(L) \cup S\left(L^{*}\right)\right)$ generates $Q(E)^{*}$ as a vector space. 
The following was proved in [BM89, item 3.10].

Theorem 10.4. Every dual-critical pair is dual-perfect.

Note that a compact, oriented Riemannian manifold for which equality holds in (9.2) is necessarily connected.

Proposition 10.5. Let $(X, g)$ be a compact, oriented Riemannian $n$ manifold with positive first Betti number $b=b_{1}(X, \mathbb{R})$. Assume that

$$
\operatorname{confsys}_{1}(g) \operatorname{sys}_{n-1}(g)=\gamma_{b}^{\prime} \operatorname{vol}_{n}(g)^{\frac{n-1}{n}} .
$$

Then every harmonic one-form on $(X, g)$ has constant norm.

Proof. By Hodge theory, the space $E$ of harmonic 1-forms on $X$ endowed with the $L^{2}$ scalar product is canonically isometric to the space $\left(H^{1}(X, \mathbb{R}),\|\|_{2}^{*}\right)$. In $E$ we have the pair of dual lattices $L$ and $L^{*}$ that correspond to $H^{1}(X, \mathbb{Z})_{\mathbb{R}}$ and $I\left(H_{1}(X, \mathbb{Z})_{\mathbb{R}}\right)$.

In the terminology of [BM89], our assumption says that the pair $\left(L, L^{*}\right)$ is dual-critical. By Theorem 10.4, the pair $\left(L, L^{*}\right)$ is dual-perfect in the sense of Definition 10.3. In our situation, we have a canonical map $p: X \rightarrow Q(E)$, $x \mapsto p_{x}$, defined by

$$
p_{x}(\alpha, \beta)=\langle\alpha(x), \beta(x)\rangle
$$

for $\alpha, \beta \in E$. By Lemma 10.2, every $\gamma \in S(L) \cup S\left(L^{*}\right)$ is a harmonic form of constant norm. This means that $\varphi(\gamma) \in Q(E)^{*}$ is constant on the image $p(X) \subseteq Q(E)$ of $p$. Since the elements $\varphi(\gamma), \gamma \in S(L) \cup S\left(L^{*}\right)$ generate $Q(E)^{*}$, every element of $Q(E)^{*}$ is constant on $p(X)$. In particular, if $\gamma \in E$ then

$$
\varphi(\gamma)\left(p_{x}\right)=p_{x}(\gamma, \gamma)=|\gamma(x)|^{2}
$$

does not depend on $x \in X$.

Remark 10.6. In terms of matrix traces, the argument above can be paraphrased as follows. The vectors $s \in S(L) \cup S\left(L^{*}\right)$ give rise to rank 1 symmetric matrices $s s^{t} \in \operatorname{Sym}\left(\mathbb{R}^{b}\right)$. A quadratic form $q_{A}(x)$ defined by a symmetric matrix $A$ gives rise to a linear map $Q: \operatorname{Sym}\left(\mathbb{R}^{b}\right) \rightarrow \mathbb{R}$ by the formula

$$
Q\left(x x^{t}\right)=q_{A}(x)=x^{t} A x=\operatorname{Tr}\left(x^{t} A x\right)=\operatorname{Tr}\left(A x x^{t}\right) .
$$

Therefore, the map $Q$ is uniquely determined by its values on the elements $s s^{t} \in \operatorname{Sym}\left(\mathbb{R}^{b}\right)$, which form a spanning set by dual-perfection. It follows that every harmonic 1 -form on $X$ has constant norm. 
Remark 10.7. The following question was asked by A. Katok in 2002. Does there exist an example of a Riemannian metric with a pair of harmonic oneforms of constant norm, whose pointwise scalar product is not constant? Such an example is, in fact, constructed in [NV03], showing that the BergéMartinet criterion (Theorem 10.4) cannot be bypassed in the proof of Theorem 1.2.

\section{Proof of Theorem 1.2.}

Assume first that there exists a Riemannian submersion $F$ with connected, minimal fibers of a compact, oriented, $n$-dimensional Riemannian manifold $(X, g)$, with first Betti number $b \geq 1$, onto a flat torus $\mathbb{R}^{b} / L$ where $L$ denotes a lattice in Euclidean space $\left(\mathbb{R}^{b},||\right)$. We will show that in such a situation, we necessarily have

$$
\begin{aligned}
\operatorname{stsys}_{1}(g) & =\lambda_{1}(L), \\
\operatorname{confsys}_{1}(g) & =\lambda_{1}(L) \operatorname{vol}_{n}(g)^{-\frac{1}{n}} \\
\operatorname{sys}_{n-1}(g) & =\lambda_{1}\left(L^{*}\right) \operatorname{vol}_{n}(g) .
\end{aligned}
$$

If $L$ is dual-critical, formulas (11.1) imply that $\operatorname{stsys}_{1}(g) \operatorname{sys}_{n-1}(g)=$ $\gamma_{b}^{\prime} \operatorname{vol}_{n}(g)$ and confsys $_{1}(g)$ sys $_{n-1}(g)=\gamma_{b}^{\prime} \operatorname{vol}_{n}(g)^{\frac{n-1}{n}}$. First note that the harmonic one-forms on $X$ are precisely the pull-backs of the constant one-forms on $\mathbb{R}^{b} / L$, and hence all of them have constant norm, $c f$. Proposition 6.3. Now Corollaries 8.2 and 8.3 imply that, up to the factor $\operatorname{vol}_{n}(g)^{\frac{1}{p}-\frac{1}{2}}$, the $L^{p}$-norms on $H_{1}(X, \mathbb{R})$ and on $H_{n-1}(X, \mathbb{R})$ coincide with the $L^{2}$-norms \|\|$_{2}$. In particular, we have

$$
\operatorname{stsys}_{1}(g)=\lambda_{1}\left(H_{1}(X, \mathbb{Z})_{\mathbb{R}},\|\|_{2}\right) \operatorname{vol}_{n}(g)^{\frac{1}{2}}
$$

and

$$
\operatorname{confsys}_{1}(g)=\operatorname{stsys}_{1}(g) \operatorname{vol}_{n}(g)^{-\frac{1}{n}}
$$

and furthermore,

$$
\operatorname{sys}_{n-1}(g)=\lambda_{1}\left(H_{n-1}(X, \mathbb{Z})_{\mathbb{R}},\|\|_{2}\right) \operatorname{vol}_{n}(g)^{\frac{1}{2}} .
$$

By Remark 7.2, the Poincaré duality map

$$
P D_{\mathbb{R}}: H^{1}(X, \mathbb{R}) \rightarrow H_{n-1}(X, \mathbb{R})
$$

preserves the $L^{2}$-norms. Since it maps $H^{1}(X, \mathbb{Z})_{\mathbb{R}}$ onto $H_{n-1}(X, \mathbb{Z})_{\mathbb{R}}, c f$. [BK03, Section 3], equation (11.4) is equivalent to

$$
\operatorname{sys}_{n-1}(g)=\lambda_{1}\left(H^{1}(X, \mathbb{Z})_{\mathbb{R}},\|\|_{2}^{*}\right) \operatorname{vol}_{n}(g)^{\frac{1}{2}} .
$$


Since the fibers of the submersion $F$ are connected, the induced homomorphism $\pi_{1}(X) \rightarrow \pi_{1}\left(\mathbb{R}^{b} / L\right)$ is onto, and hence we obtain an epimorphism $H_{1}(X, \mathbb{Z}) \rightarrow H_{1}\left(\mathbb{R}^{b} / L, \mathbb{Z}\right)$. Since $b=b_{1}(X, \mathbb{R})$, the epimorphism $F_{*}: H_{1}(X, \mathbb{Z})_{\mathbb{R}} \rightarrow H_{1}\left(\mathbb{R}^{b} / L, \mathbb{Z}\right)_{\mathbb{R}}$ is an isomorphism. By duality, the map $F^{*}: H^{1}\left(\mathbb{R}^{b} / L, \mathbb{Z}\right)_{\mathbb{R}} \rightarrow H^{1}(X, \mathbb{Z})_{\mathbb{R}}$ is an isomorphism. Now $H^{1}\left(\mathbb{R}^{b} / L, \mathbb{Z}\right)_{\mathbb{R}}$ can be identified with the dual lattice, $L^{*}$. An element $\theta \in L^{*}$ satisfies

$$
\left\|F^{*} \theta\right\|_{2}^{*}=|\theta| \operatorname{vol}_{n}(g)^{\frac{1}{2}} .
$$

Hence, equations (11.2), (11.3), and (11.5) imply our claim, equation (11.1).

Conversely, we assume that $\operatorname{confsys}_{1}(g)$ sys $_{n-1}(g)=\gamma_{b}^{\prime} \operatorname{vol}_{n}(g)^{\frac{n-1}{n}}$. Then Proposition 10.5 implies that every harmonic one-form on $X$ has constant norm. Integrating harmonic one-forms that form a basis for the integer lattice in $H^{1}(X, \mathbb{R})$, we obtain a Riemannian submersion $F$ with minimal fibers from $(X, g)$ onto a flat torus $\mathbb{R}^{b} / L, c f$. (6.3). Since we obtain $F$ from a basis of the integer lattice $H^{1}(X, \mathbb{Z})_{\mathbb{R}}$, we see that

$$
F^{*}: H^{1}\left(\mathbb{R}^{b} / L, \mathbb{Z}\right)_{\mathbb{R}} \rightarrow H^{1}(X, \mathbb{Z})_{\mathbb{R}}
$$

is an isomorphism. It follows that $F$ induces an epimorphism at the level of fundamental groups, and hence the fibers of $F$ are connected. Note that the first part of the proof implies the identities $\operatorname{confsys}_{1}(g)=\lambda_{1}(L) \operatorname{vol}_{n}(g)^{-\frac{1}{n}}$ and $\operatorname{sys}_{n-1}(g)=\lambda_{1}\left(L^{*}\right) \operatorname{vol}_{n}(g)$. Hence $\lambda_{1}(L) \lambda_{1}\left(L^{*}\right)=\gamma_{b}^{\prime}$, and therefore $L$ is dual-critical.

\section{Construction of all extremal metrics.}

In this section we will present a construction that starts with a submersion $F: X \rightarrow T^{m}$ of a compact manifold $X$ to a flat torus, and provides all Riemannian metrics on $X$ for which $F$ is a Riemannian submersion with minimal fibers. If $X$ admits a submersion to $T^{b}$, where $b=b_{1}(X, \mathbb{R})$, this construction provides all metrics on $X$ for which all harmonic one-forms have constant norm, cf. Proposition 6.3.

In this construction, we can prescribe arbitrary metrics of fixed volume on the fibers of $F$. This does not determine the metric on $X$ uniquely. We additionally have to define a horizontal distribution $\mathcal{H}$ transverse to the vertical distribution $\mathcal{V}=\operatorname{ker}(d F)$. The choice of $\mathcal{H}$ is restricted by the requirement that the horizontal lift of every flow on the base has to preserve the volume elements of the fibers. The necessity of this condition follows from the requirement that all fibers be minimal, $c f$. Lemma 12.3. We start by providing a multiparameter variant of J. Moser's theorem [Mo65] on the 
existence of volume-preserving diffeomorphisms. This will allow us to find the horizontal distributions, starting with an arbitrary family of metrics on the fibers of constant volume.

We first explain the setting and fix some notation. Let $F: X \rightarrow Y$ denote a submersion between compact, connected and oriented manifolds $X$ and $Y$.

Definition 12.1. Let $\mathcal{X}_{F}$ denote the set of projectable vector fields on $X$. Namely, a vector field $V$ on $X$ is in $\mathcal{X}_{F}$ if there exists a vector field $W$ on $Y$ such that $D F \circ V=W \circ F$.

Such a vector field $W$ on $Y$ will be called the projection of $V \in \mathcal{X}_{F}$ and it will be denoted by $W=F_{*}(V)$. In the literature on foliations, projectable vector fields are also called "basic" or "foliate". Note that $\mathcal{X}_{F}$ is a Lie subalgebra of the Lie algebra of all vector fields on $X$. Furthermore, $F_{*}$ is a homomorphism from the Lie algebra $\mathcal{X}_{F}$ to the Lie algebra $\mathcal{X}(Y)$ of all vector fields on $Y$. In terms of the flow, $\Phi$, of $V \in \mathcal{X}_{F}$, and the flow, $\Psi$, of $F_{*}(V)=W$, the relation $D F \circ V=W \circ F$ is equivalent to

$$
F \circ \Phi_{t}=\Psi_{t} \circ F \quad \text { for all } t \in \mathbb{R} .
$$

In particular, if $y \in Y$ then $\Phi_{t}$ maps the fiber $F^{-1}(y)$ to the fiber $F^{-1}\left(\Psi_{t}(y)\right)$. We denote the diffeomorphism $\Phi_{t} \mid F^{-1}(y)$ by $\Phi_{t, y}$.

In addition, we assume that on every fiber $F^{-1}(y)$, we are given a volume element $\alpha^{y} \in \Omega^{n-m}\left(F^{-1}(y)\right)$, where $\operatorname{dim} X=n$ and $\operatorname{dim} Y=m$, that varies smoothly with $y \in Y$. We say that the flow $\Phi$ of $V \in \mathcal{X}_{F}$ preserves $\alpha=$ $\left(\alpha^{y}\right)_{y \in Y}$ if

$$
\Phi_{t, y}^{*} \alpha^{\psi_{t}(y)}=\alpha^{y}
$$

for all $(t, y) \in \mathbb{R} \times Y$. We denote by $\mathcal{X}(\alpha)$ the Lie subalgebra of all projectable vector fields whose flows preserve $\alpha$.

Proposition 12.2. The homomorphism $F_{*}: \mathcal{X}(\alpha) \rightarrow \mathcal{X}(Y)$ is an epimorphism of Lie algebras.

This multiparameter version of J. Moser's theorem [Mo65] can be proved along the lines of the original proof in [Mo65].

Now we specialize the preceding discussion to the case of a Riemannian submersion $F: X \rightarrow Y$ between Riemannian manifolds $X$ and $Y$, where $\operatorname{dim} X=n, \operatorname{dim} Y=m$. Actually, the discussion is purely local. Every flow $\Psi$ on $Y$ has a unique horizontal lift $\Phi$ to $X$, i.e. $\Phi$ is the flow on $X$ whose flow lines are the horizontal lifts of the flow lines of $\Psi$. Equivalently, if 
$\Psi$ is the flow of the vector field $W$ on $Y$, then $\Phi$ is the flow of the horizontal lift $V$ of $W$ defined by requiring that $V \in \mathcal{X}_{F}, F_{*}(V)=W$ and that $V$ is everywhere horizontal, i.e. orthogonal to the vertical distribution $\mathcal{V}=$ $\operatorname{ker}(d F)$. Obviously, the horizontal lift of any flow on $Y$ maps fibers of $F$ to fibers of $F$.

Lemma 12.3. Suppose $F: X \rightarrow Y$ is a Riemannian submersion between oriented Riemannian manifolds. Then all the fibers of $F$ are minimal submanifolds if and only if the horizontal lift of every flow on $Y$ maps the volume elements of the fibers of $F$ to each other.

Proof. This follows from [Ru79, Proposition 1].

Remark 12.4. Actually, the "if"-part in Lemma 12.3 is true under the following seemingly weaker assumption. For every $y \in Y$ there are $m$ vector fields on $Y$ that are linearly independent at $y$ and whose flows have horizontal lifts that preserve the volume elements of the fibers of $F$.

Let us now assume that $F$ is a submersion of a compact, oriented and connected $n$-manifold $X$ onto a flat torus $\mathbb{R}^{m} / L$ where $L$ is a lattice in Euclidean space $\mathbb{R}^{m}$. Moreover, we assume that for all $y \in \mathbb{R}^{m} / L$, we are given a volume element $\alpha^{y}$ on $F^{-1}(y)$ that depends smoothly on $y$.

We let $\mathcal{X}_{c}(\alpha)$ denote the set of all vector fields $V \in \mathcal{X}(\alpha)$ such that

$$
d F(V)=\left(d F_{1}(V), \ldots, d F_{m}(V)\right) \text { is a constant vector in } \mathbb{R}^{m} .
$$

Obviously $\mathcal{X}_{c}(\alpha)$ is a linear subspace of $\mathcal{X}(\alpha)$ and

$$
l_{F}: \mathcal{X}_{c}(\alpha) \rightarrow \mathbb{R}^{m}, l_{F}(V)=d F(V),
$$

is a linear map. Proposition 12.2 implies

$$
l_{F}: \mathcal{X}_{c}(\alpha) \rightarrow \mathbb{R}^{m} \text { is onto. }
$$

To define a horizontal distribution $\mathcal{H}$, we will choose a linear right inverse $H: \mathbb{R}^{m} \rightarrow \mathcal{X}_{c}(\alpha)$ to $l_{F}$ and set

$$
\mathcal{H}_{x}=\left\{\left.H(v)\right|_{x} \mid v \in \mathbb{R}^{m}\right\} \subseteq T X_{x}
$$

for $x \in X$. Note that we have much freedom in the choice of $H$ if $n-m>1$. The kernel of $l_{F}$ consists of those vertical vector fields $V$ that preserve each $\alpha^{y}$, i.e. on every fiber $F^{-1}(y)$ one has a vector field $V^{y}=V \mid F^{-1}(y)$ whose 
flow preserves $\alpha^{y}$ and that varies smoothly with $y \in T^{m}$. In particular, $\operatorname{ker}\left(l_{F}\right) \subseteq \mathcal{X}_{c}(\alpha)$ is an infinite-dimensional Lie algebra if $n-m>1$.

We are now in a position to describe our construction of metrics for which a given submersion onto a flat torus is Riemannian and with minimal fibers.

Construction 12.5. Let $X$ be a compact, oriented and connected $n$ manifold and $F: X \rightarrow T^{m}=\mathbb{R}^{m} / L$ a submersion onto a flat torus.

1) Choose a smooth family $g^{y}, y \in T^{m}$, of Riemannian metrics on the fibers $F^{-1}(y)$ of $F$, such that the total Riemannian volume of $\left(F^{-1}(y), g^{y}\right)$ does not depend on $y \in T^{m}$.

2) Let $\alpha=\left(\alpha_{y}\right)_{y \in Y}$ denote the family of Riemannian volume forms on the fibers of $F$. Choose a linear right inverse $H: \mathbb{R}^{m} \rightarrow \mathcal{X}_{c}(\alpha)$ to $l_{F}$ and define the subbundle $\mathcal{H} \subseteq T X$ by

$$
\mathcal{H}_{x}=\left\{\left.H(v)\right|_{x} \mid v \in \mathbb{R}^{m}\right\}
$$

for $x \in X$.

3) Now define a Riemannian metric $g$ on $X$ by requiring:

(a) The subbundles $\mathcal{V}=\operatorname{ker}(d F)$ and $\mathcal{H}$ are orthogonal to each other.

(b) For all $x \in X$ the isomorphism $d F_{x} \mid \mathcal{H}_{x}: \mathcal{H}_{x} \rightarrow \mathbb{R}^{m}$ is an isometry.

(c) For all $y \in T^{m}$ the inclusion $\left(F^{-1}(y), g^{y}\right) \rightarrow(X, g)$ is isometric.

Theorem 12.6. The Riemannian metrics $g$ on $X$ arising from Construction 12.5 are precisely those for which $F: X \rightarrow T^{m}=\mathbb{R}^{m} / L$ is a Riemannian submersion with minimal fibers.

Proof. Suppose $g$ arises from the construction. Then properties (a) and (b) imply that $F$ is a Riemannian submersion. The vector fields $V_{i}=H\left(e_{i}\right)$, $1 \leq i \leq m$, form an orthonormal frame field for $\mathcal{H}=\mathcal{V}^{\perp}$. Because of (c) and $\mathcal{X}_{c}(\alpha) \subseteq \mathcal{X}(\alpha)$, their flows preserve the volume elements induced by $g$ on the fibers. This implies that the fibers are minimal submanifolds of $(X, g)$, cf. Remark 12.4. Conversely, suppose $g$ is a Riemannian metric on $X$ such that $F: X \rightarrow \mathbb{R}^{m} / L$ is a Riemannian submersion with minimal fibers. We describe how the metrics on the fibers and the map $H$ have to be chosen so that our construction yields $g$. For the metrics $g^{y}, y \in \mathbb{R}^{m} / L$, on the fibers we must obviously take the metrics induced by $g$. If $v \in \mathbb{R}^{m}$ let $\bar{\theta}$ be the constant one-form on $\mathbb{R}^{m} / L$ that is dual to $v$ with respect to the scalar 
product on $\mathbb{R}^{m}$ and let $V$ be $g$-dual to $F^{*} \bar{\theta}$. Now Lemma 12.3 implies that $V \in \mathcal{X}(\alpha)$, where $\alpha=\left(\alpha_{y}\right)_{y \in Y}$ denotes the volume elements induced by $g$ on the fibers. Moreover, $l_{F}(V)=v$, i.e. the (linear) map that maps $v \in \mathbb{R}^{m}$ to $V \in \mathcal{X}_{c}(\alpha)$ is a right inverse to $l_{F}$ which we take to be $H$. Then the horizontal distribution $\mathcal{H}$ induced by $H$ is $g$-orthogonal to $\mathcal{V}=\operatorname{ker}(d F)$ and our construction applied to these choices of $g^{y}, y \in \mathbb{R}^{m} / L$, and $H$ yields the metric $g$.

Corollary 6.5 shows to what extent our construction leads to nonisometric metrics $g$, and how it depends on the submersion $F$ and the flat torus $\mathbb{R}^{m} / L$.

If $X$ admits a metric such that all harmonic one-forms have constant norm, then there exists a submersion $F: X \rightarrow T^{b}$ for $b=b_{1}(X, \mathbb{R})$, cf. Proposition 6.3. Starting from such submersions $F$ and from arbitrary flat metrics on $T^{b}$, our construction yields all metrics on $X$ for which all harmonic one-forms have constant norm.

Similarly, $X$ can support metrics $g$ for which equality holds in (1.1) only if $X$ admits a submersion onto $T^{b}$ for $b=b_{1}(X, \mathbb{R})$. Starting from such submersions and from flat metrics on $T^{b}$ coming from dual-critical lattices $L \subseteq \mathbb{R}^{b}$, the construction yields all metrics on $X$ satisfying equality in (1.1).

\section{Acknowledgments.}

We are grateful to D. Ebin, E. Leichtnam, and J. Lafontaine for discussions concerning the smooth dependence on parameters in Moser's method, $c f$. Proposition 12.2. We thank E. Kuwert for providing references concerning closed forms minimizing the $L^{p}$-norm in their cohomology class.

\section{References.}

[Bab02] Babenko, I.: Forte souplesse intersystolique de variétés fermées et de polyèdres. Annales de l'Institut Fourier 524 (2002), 12591284 .

[BabK98] Babenko, I.; Katz, M.: Systolic freedom of orientable manifolds, Annales Scientifiques de l'E.N.S. (Paris) 31 (1998), 787-809.

[BKS98] Babenko, I.; Katz, M.; Suciu, A.: Volumes, middle-dimensional systoles, and Whitehead products. Math. Res. Lett. 5 (1998), no. 4, 461-471. 
[BCIK1] Bangert, V.; Croke, C.; Ivanov, S.; Katz, M.: Filling area conjecture and ovalless real hyperelliptic surfaces, preprint. arXiv:math.DG/0405583

[BCIK2] Bangert, V.; Croke, C.; Ivanov, S.; Katz, M.: Boundary case of equality in optimal Loewner-type inequalities, preprint. arXiv:math.DG/0406008

[BK03] Bangert, V.; Katz, M.: Stable systolic inequalities and cohomology products. Comm. Pure Appl. Math. 56 (2003), 979-997. math.DG/0204181

[Bav86] Bavard, C.: Inégalité isosystolique pour la bouteille de Klein, Math. Ann. 274 (1986), no. 3, 439-441.

[BM89] Bergé, A.-M.; Martinet, J.: Sur un problème de dualité lié aux sphères en géométrie des nombres. J. Number Theory 32, 14-42 (1989).

[BS94] Buser, P.; Sarnak, P.: On the period matrix of a Riemann surface of large genus. With an appendix by J. H. Conway and N. J. A. Sloane. Invent. Math. 117 (1994), no. 1, 27-56.

[Ch93] Chavel, I.: Riemannian geometry - a modern introduction. Cambridge Tracts in Mathematics, 108. Cambridge University Press, Cambridge, 1993.

[CS94] Conway, J. H.; Sloane, N. J. A.: On lattices equivalent to their duals. J. Number Theory 48 (1994), no. 3, 373-382.

[CK03] Croke, C.; Katz, M.: Universal volume bounds in Riemannian manifolds, Surveys in Differential Geometry VIII, Lectures on Geometry and Topology held in honor of Calabi, Lawson, Siu, and Uhlenbeck at Harvard University, May 3- 5, 2002, edited by S.T. Yau (Somerville, MA: International Press, 2003.) pp. 109 137. See arXiv:math.DG/0302248

[ES64] Eells, J.; Sampson, J. H.: Harmonic mappings of Riemannian manifolds. Amer. J. Math. 86 (1964) 109-160.

[Fe69] Federer, H.: Geometric Measure Theory. Springer 1969.

[Fe74] Federer, H.: Real flat chains, cochains, and variational problems. Indiana Univ. Math. J. 24, 351-407 (1974). 
[Fr99] Freedman, M.: $\mathbf{Z}_{2}$-systolic-freedom. Proceedings of the Kirbyfest (Berkeley, CA, 1998), 113-123. Geometry and Topology Monographs, 2. Geometry and Topology, Institute of Mathematics, Warwick University, Coventry 1999.

[Gr83] Gromov, M.: Filling Riemannian manifolds. J. Differential Geom. 18, 1-147 (1983).

[Gr96] Gromov, M.: Systoles and intersystolic inequalities. In: Actes de la Table Ronde de Géométrie Différentielle (Luminy 1992), 291362. Séminaires et Congrès, 1. Soc. Math. France, Institut Henri Poincaré, Paris 1996.

[Gr99] Gromov, M.: Metric structures for Riemannian and nonRiemannian spaces. Progress in Mathematics 152. Birkhäuser, Boston 1999.

[Ha92] Hamburger, C.: Regularity of differential forms minimizing degenerate elliptic functionals. J. Reine Angew. Math. 431 (1992), $7-64$.

[He86] Hebda, J.: The collars of a Riemannian manifold and stable isosystolic inequalities. Pacific J. Math. 121, 339-356 (1986).

[IK04] Ivanov, S.; Katz, M.: Generalized degree and optimal Loewnertype inequalities, Israel J. Math. 141 (2004), 221-233.

[KT82] Kamber, F.W., Tondeur, P.: Harmonic foliations. In: Harmonic maps (edited by R.J. Knill, M. Kalka, H.C.J. Sealey), 87-121. Lecture Notes in Math. 949, Springer 1982.

[Ka95] Katz, M.: Counterexamples to isosystolic inequalities. Geom. Dedicata 57 (1995), 195-206.

[Ka02] Katz, M.: Local calibration of mass and systolic geometry. Geometric and Functional Analysis 12, issue 3 (2002), 598-621.

[Ka03] Katz, M.: Four-manifold systoles and surjectivity of period map, Comment. Math. Helvetici, 78 (2003) 772-876. arXiv:math.DG/0302306

[KL04] Katz, M.; Lescop, C.: Filling area conjecture, optimal systolic inequalities, and the fiber class in abelian covers, preprint. 
[KS04] Katz, M.; Sabourau, S.: Hyperelliptic surfaces are Loewner, preprint.

[KN63] Kobayashi, S.; Nomizu, K.: Foundations of Differential Geometry I. Interscience, New York 1963.

[Ko01] Kotschick, D.: On products of harmonic forms. Duke Math. J. 107 (2001), no. 3, 521-531.

[LLS90] Lagarias, J.C.; Lenstra, H.W., Jr.; Schnorr, C.P.: Bounds for Korkin-Zolotarev reduced bases and successive minima of a lattice and its reciprocal lattice. Combinatorica 10, 343-358 (1990).

[Li69] Lichnerowicz, A.: Applications harmoniques dans un tore. C.R. Acad. Sci., Sér. A, 269, 912-916 (1969).

[MH73] Milnor, J.; and Husemoller, D.: Symmetric bilinear forms. Springer, 1973.

[Mo65] Moser, J.: On the volume elements on a manifold. Transactions Amer. Math. Soc. 120, 286-294 (1965).

[NV03] Nagy, P. A.; Vernicos, C.: The length of harmonic forms on a compact Riemannian manifold. Transactions A. M. S. (to appear). arXiv:math.DG/0301369

[Pa01] Paternain, G. P.: Schrödinger operators with magnetic fields and minimal action functionals. Israel J. Math. 123 (2001), 1-27.

[Pu52] Pu, P. M.: Some inequalities in certain nonorientable Riemannian manifolds. Pacific J. Math. 2, (1952), 55-71.

[Ru79] Rummler, H.: Quelques notions simples en géométrie riemannienne et leurs applications aux feuilletages compacts. Comment. Math. Helv. 54 (1979), 224-239.

[Se88] Sergiescu, V.: Basic cohomology and tautness of Riemannian foliations. Appendix B in: P. Molino, Riemannian foliations, 235248. Progress in Math. 73, Birkhäuser, Boston-Basel 1988.

[Si70] Sibner, L. M.; Sibner, R. J.: A non-linear Hodge-de-Rham theorem. Acta Math. 125 (1970) 57-73.

[Sa88] Sakai, T.: A proof of the isosystolic inequality for the Klein bottle. Proc. Amer. Math. Soc. 104 (1988), no. 2, 589-590. 
[Su79] Sullivan, D.: A homological characterization of foliations consisting of minimal surfaces. Comment. Math. Helv. 54 (1979), $218-223$.

VICTOR BANGERT

Mathematisches Institut

UNIVERSITÄT FREIBURG

ECKerstr. 1, 79104 Freiburg, Germany

bangert@mathematik. uni-freiburg.de

Mikhail KatZ

Department of Mathematics and Statistics

Bar Ilan University, Ramat Gan 52900, IsRael

katzmik@math.biu.ac.il

RECEIVED APRIL 2003. 